\title{
Article \\ A Wireless Animal Robot Stimulation System Based on Neuronal Electrical Signal Characteristics
}

\author{
Rui Yan ${ }^{1,2,+}$, Zhaozhen Chen ${ }^{1,2,+}$,Hui Wang ${ }^{1,2}$,Junqing Yang ${ }^{1,2}$, Yong Shi ${ }^{1,2}$, \\ Haoran Zhu ${ }^{1,2}$,Pingqiu Zhang ${ }^{1,2}$,Yangyang Liu ${ }^{1,2}$ and Ruituo Huai 1,2,* \\ 1 College of Electrical and Automation Engineering, Shandong University of Science and Technology, \\ Qingdao, PR China; 876937096@qq.com(R.Y.) \\ 2 Shandong Robotics and Intelligent Technology Key Laboratory, Qingdao, PR China; \\ huairuituo@163.com(R.H.) \\ * Correspondence: huairuituo@163.com(R.H.); Tel.: +86-1596-680-3873 (R.H.) \\ + These authors contributed equally to this work.
}

\begin{abstract}
As a stimulus signal, coded electrical signals can control the motion behavior of animals, which has been widely used in the field of animal robots. In current research, most of the stimulus signals used by researchers are traditional waveforms, such as square waves. To enrich the stimulus waveform, a wireless animal robot stimulation system based on neuronal electrical signal characteristics is presented in this paper. The stimulator uses the CC1101 wireless module to control animal behavior through brain stimulation. The LabVIEW-based graphical user interface(GUI) can manipulate brain stimulation remotely while the stimulator powered by battery. Additionally, The spikes of animals have been simulated by this system through Direct Digital Synthesizer(DDS) algorithm. The GUI enable users to customize the combination of these analog spike signals. The recombined signals are sent to the stimulator through CC1101 as stimulus signals. In vivo experiments conducted on five pigeons verified the efficacy of the stimulation mechanism. The analog spike signal with an amplitude of 3-5V successfully caused the pigeon's turning behavior. The feasibility of the analog spike signals as stimulus signals was successfully verified. Increased the diversity of stimulus waveforms in the field of animal robots.
\end{abstract}

Keywords: animal robots; neuronal electrical signal; electrical stimulation; Direct Digital Synthesis algorithm

\section{Introduction}

Animal robots are new types of robots that directly control animal movements through human intervention in neural circuit activities. Compared with traditional robot technology, animal robots can rely on the motion functions of biological carriers without carrying additional energy drives. It has shown great advantages in environmental adaptability, concealment, mobility, load capacity, etc[1,2]. Animal robots has important theoretical and applied value in the fields of neuroscience and engineering, disaster rescue, animal behavior, etc.[3-5].

Beginning in the 1990s, researchers from various countries have begun research on the use of biological control technology to develop animal robots. For example, the University of Tokyo in Japan conducted related experiments on cockroaches[6]. The researchers chose to apply electrical stimulation to the best position to control their movement to achieve control, and finally successfully controlled the cockroach to walk straight. In 2006, New Scientist magazine reported that the atema research team of Boston University completed the "Shark Agent" study[7]. They implanted microelectrodes in the olfactory center of the Squalus acanthias's brain to generate virtual odor stimuli by electrically stimulating the olfactory center, which induces the shark to swim with odor tracking. In 2007, Su Xuecheng et al. were the first researchers who completed the pigeon 
navigation produsure[8]. They used specially processed insulated electrodes to directly stimulate the fear-sensing nucleus to control the pigeon. Finally they were able to control the pigeon to complete take-off, landing, turning and other actions. In 2009, researchers at Hiroshima University chose to stimulate the medial longitudinal fasciculus to achieve effective control of goldfish[9]. In 2015, Erickson and others from the Department of Physical Engineering of Washington and Lee University found effective parameters for stimulating cockroaches. The stimulus waveform was bipolar pulse, which realized the behavior of cockroaches moving forward and leftward[10]. In 2017, researches in Seoul National University selected new materials to make electrodes. They chose liquid crystal polymer as the outsourcing material for implantation experiments. They used biphasic current stimulation to finally control the behavior of the pigeons[11]. In 2019, A handheld neural stimulation controller was proposed in Seoul National University for bird remote navigation. This system consists of a handheld neural stimulation controller and a fully-implanted stimulator[12]. In the same year, they applied this device to rats and achieved good results[13].

At present, most of the stimulus signals are regular waveforms such as square waves[14-17]. And in each experiment, the stimulus waveform used by the researcher will not change. The long-term use of this simple stimulation mode will produce tolerance in animals, which will greatly reduce the stimulation effect[18-23]. Hence, a wireless animal robot stimulation system based on neuronal electrical signal characteristics was proposed in this paper. The overall schematic diagram of the system is shown in Figure 1

This proposed stimulation system consists of a hardware platform based on STM32, including the signal transmitting base station and the electronic backpack, and a set of GUI. The stimulus commands generated on the GUI are wirelessly sent to the electronic backpack by the transmitting base station. The electronic backpack is carried on the animal and delivers electrical stimulus to electrodes inserted into the animal's brain.

This paper focuses on simulating animal spikes and increasing the diversity of stimulation patterns. So the DDS algorithm is used in the electronic backpack to decode the stimulation instructions and reconstruct the animal's analog spike signal. In GUI, we have stored different kinds of spikes collected in advance into the spike library. Users can customize the combination of spikes and generate stimulation parameters from the GUI to send to the electronic backpack. During the stimulation experiment, the user can arbitrarily adjust the stimulation train to increase the variety of stimulation waveforms.

In this paper, details of electronic backpack are demonstrated to show its fabrication methods and the process of spike simulation. The functionality of this system was evaluated in vivo in order to verify the feasibility of using spike signals as stimulus signals.

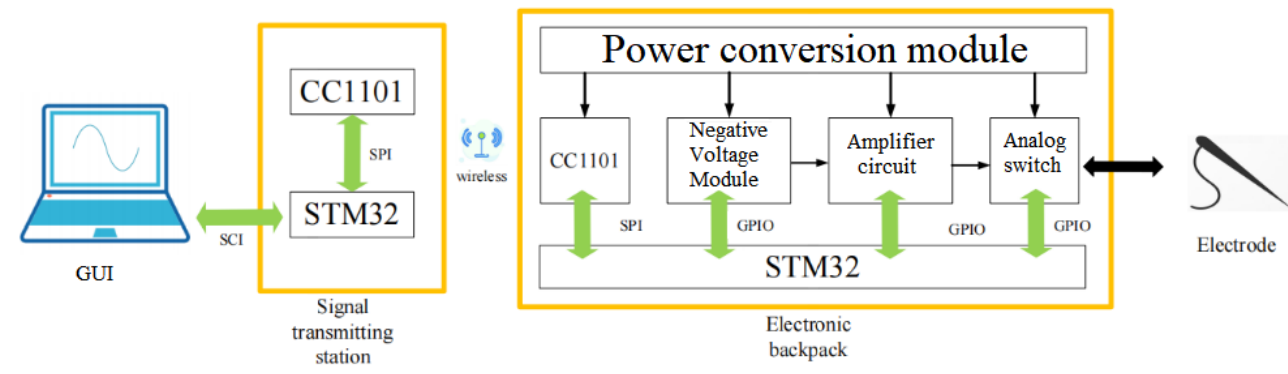

Figure 1. Overall system framework 


\section{Materials and Methods}

\subsection{Transmitting base station design}

The stimulus command is transmitted from the GUI to the transmitting base station, and then the stimulus command is wirelessly forwarded to the electronic backpack. Therefore, commercial STM32 minimum system board and CC1101 wireless module are used as signal transmitting base station. The diagram of the signal transmitting base station is shown in Figure 2.

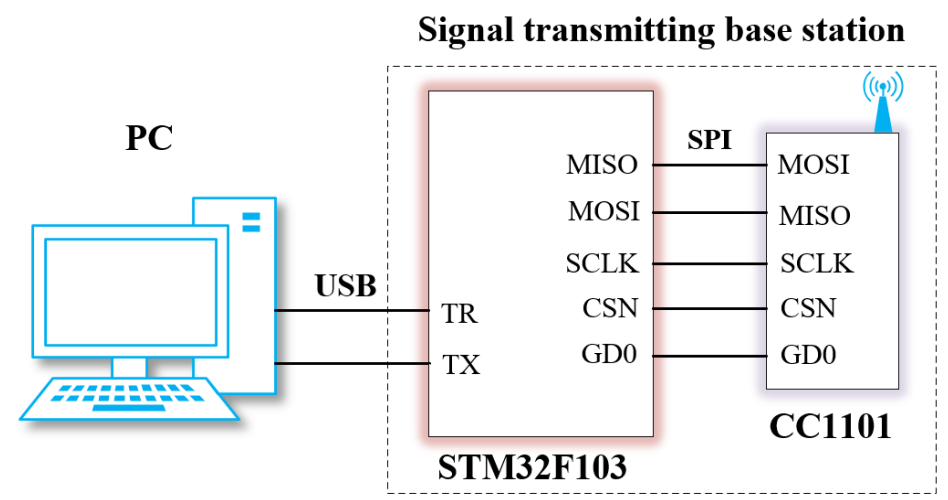

Figure 2. Signal transmitting base station

\subsection{Electronic backpack design}

Wireless module In order not to affect the normal activities of animals, the electronic backpack should use wireless transmission and be small enough. Additionally, low-power but long-range wireless communication is necessary.

To achieve these requirements, CC1101(manufactured by Texas Instruments) has been chosen for wireless communication between thetransmitting base station and the electronic backpack.Not only does CC1101 consume lower power than $\mathrm{Wi}-\mathrm{Fi}$, it can also transmit data for a longer distance (up to $100 \mathrm{~m}$ in practice) in comparison to Bluetooth.

CC1101 contains two RF ports and is a typical differential signal output. Since a monopole antenna is used for data transmission in the system design, a balun is required to be used to convert the double-ended signal into a unipolar signal. The balanced structure port is composed of two parallel wires, the current of the two wires is the same but the direction is opposite. The common mode interference is suppressed by the differential signal and then converted into a unipolar signal output by the balun[24-26]. The power consumption of the front-end amplifying part of the radio frequency chip is often the largest. Current conduction angle is reduced to reduce power consumption[27]. But reducing the current conduction angle will cause harmonic distortion, so a filter was added to solve the above problem. In this paper, the LC filter structure is selected for filtering. The LC filter structure is simple, uses fewer components, and connects the inductor in series in the path, which has better isolation and relatively better filtering effect. The wireless module circuit is shown in Figure 3.

Power conversion module A rechargeable lithium battery $(300 \mathrm{mAh}, 3.7 \mathrm{~V})$ is used to power the electronic backpack. REG710-3.3V (manufactured by Texas Instruments) is used to convert the $3.7 \mathrm{~V}$ into a stable $3.3 \mathrm{~V}$ for the MCU and CC1101. The voltage converter and multiplexer need $\pm 5 \mathrm{~V}$ power supply to output the maximum stimulus waveform of $\pm 5 \mathrm{~V}$. So REG710-5V is used as voltage boost. The size of this chip is only $2 \mathrm{~mm} \times 3 \mathrm{~mm}$, which can save a lot of circuit space. The power conversion module circuit is shown in Figure 4.

A circuit composed of TC7660 and two $10 \mu \mathrm{F}$ tantalum capacitors is used as a negative voltage generating circuit. Figure 5 shows the negative voltage generating module circuit. 


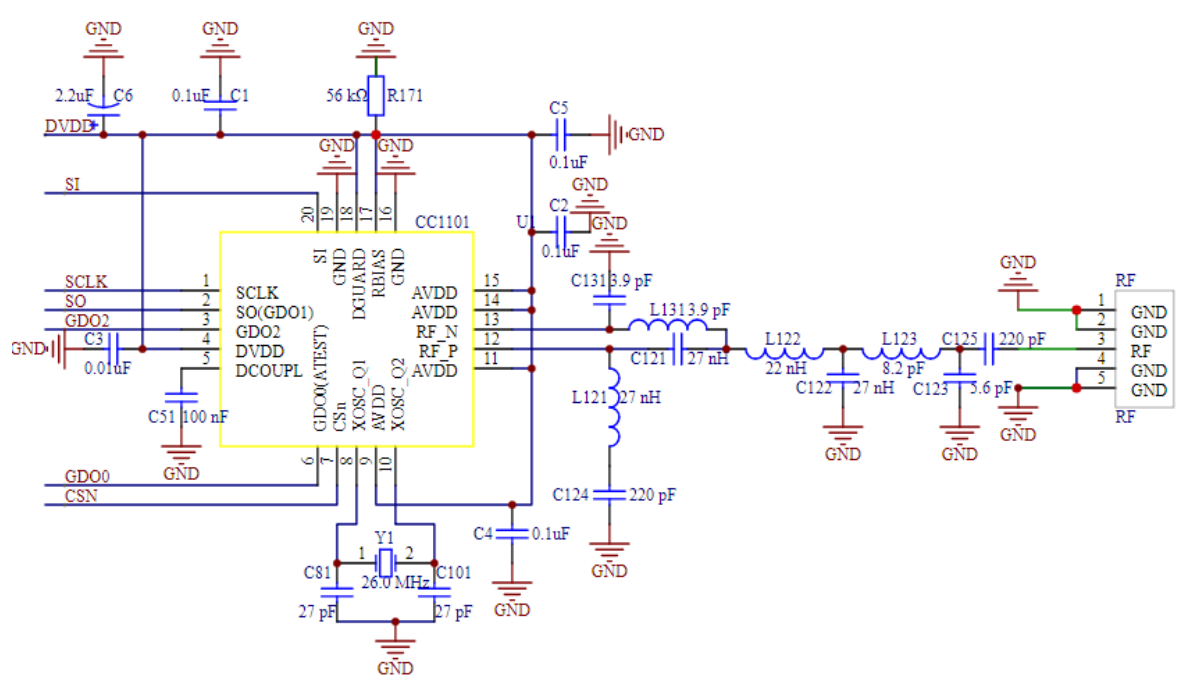

Figure 3. Schematic diagram of wireless module circuit

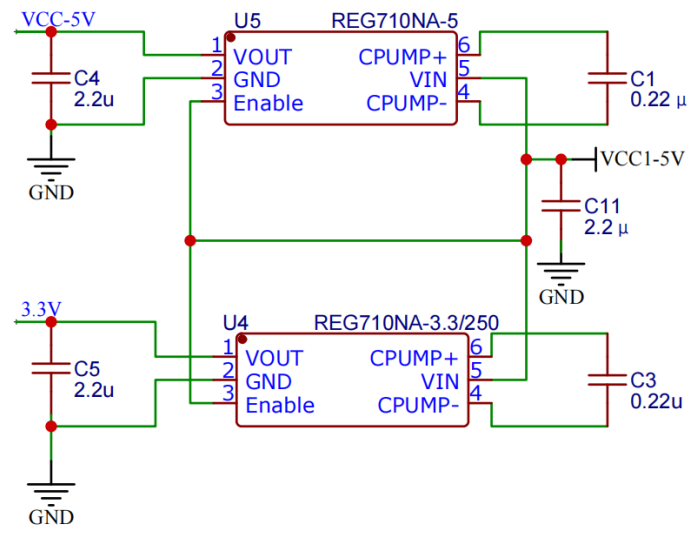

Figure 4. Power conversion module

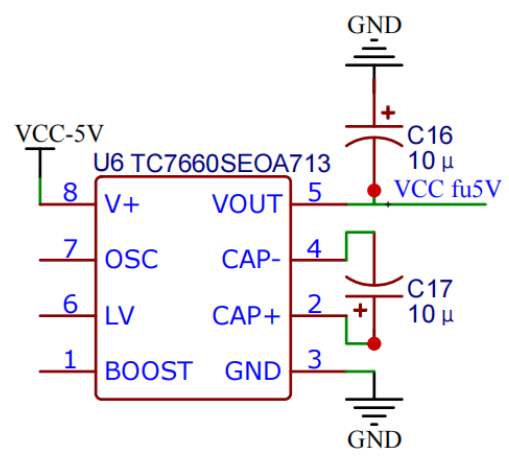

Figure 5. Negative voltage generating circuit

Biphasic pulse generating circuit The final analog spike signal to be generated by electronic backpack is a biphasic waveform. The amplitude of the output waveform is adjustable from -5 to $5 \mathrm{~V}$. So a voltage conversion circuit is designed to convert the voltage from $03.3 \mathrm{~V}$ to $-55 \mathrm{~V}$ voltage. As shown in the Figure 6 . The circuit consists of two operational amplifiers (model AD820), which are used to convert unipolar voltage to bipolar voltage and amplify bipolar voltage respectively. As shown in the Figure 6 . The output and input have the following relationship:

$$
V_{0}=\frac{R_{7}+R_{8}}{R_{7}} \times\left(\frac{R_{5}+R_{6}}{R_{5}} \times V_{\text {in }}-5 \times \frac{R_{6}}{R_{5}}\right)
$$


The electronic backpack decodes and calculates the parameters of the received analog

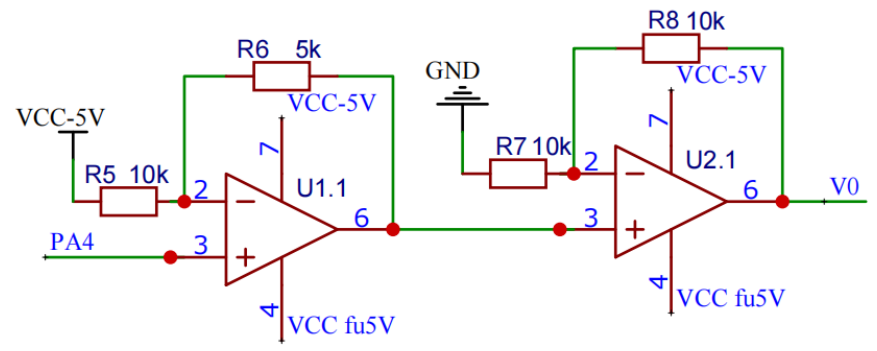

Figure 6. Biphasic pulse generating circuit

spike signal into a single-phase signal, and then outputs an ideal biphasic analog spike signal through the above-mentioned amplifying circuit.

PCB design and manufacture The schematic diagram of the circuit board design is shown in Figure 7 . The upper board size is $30 \mathrm{~mm} \times 16 \mathrm{~mm} \times 1.5 \mathrm{~mm}$, the mass is about $3.7 \mathrm{~g}$, the front is CC1101 working circuit and PCB antenna. The lower board is $38 \mathrm{~mm} \times 23 \mathrm{~mm} \times 2 \mathrm{~mm}$, the mass is about $5.5 \mathrm{~g}$, mainly including STM32F103RCT6 working circuit, a REG710 voltage conversion chip , a negative pressure generating TC7660 chip and two AD820 precision op amps. The total weight of the electronic backpack is $15.8 \mathrm{~g}$ (6.6g including battery). Two rows of $2.54 \mathrm{~mm}$ pin headers are used to connect between the two circuit boards and realize signal transmission. A rechargeable lithium battery $(300 \mathrm{mAh}, 3.7 \mathrm{~V})$ is used as a wireless power source to power the electronic backpack, and the battery is placed between the two circuit boards. The physical map of the entire electronic backpack is shown in Figure 8.

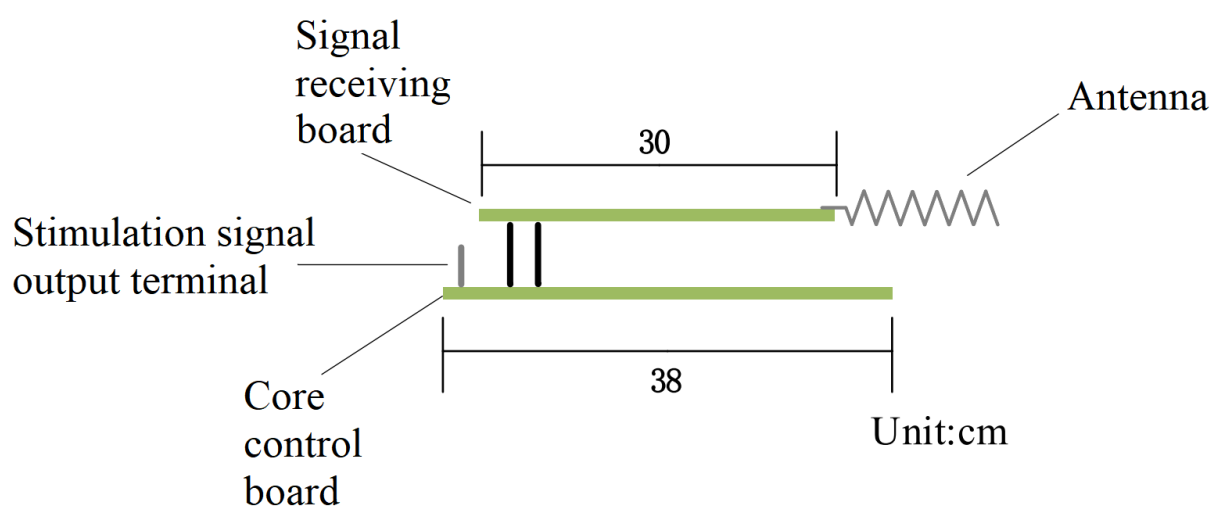

Figure 7. Schematic diagram of electronic backpack circuit board design

\subsection{Software design}

Simulating animal spike signals efficiently is our ultimate goal, so Principal Component Analysis(PCA) is used to classify pre-acquired spike data on MATLAB[28-30]. Then, the average value of each type of spike data is processed to obtain a smoother curve and the curve is stored as a spike signal. The result is shown in Figure 9.

The obtained spike potential curve needs to be fitted. The purpose of the fitting is to decompose the spike curve into multiple sinusoidal forms. In the subsequent work, the parameters obtained after fitting need to be sent to the electronic backpack through the GUI. Then, the DDS algorithm is used to process these parameters on the electronic backpack and finally output the analog spike signal. The smoothed spike No.2 as an example, and the fitting result is shown in Figure 10.

From the results of fitting, the spike No.2 is decomposed into a composite form of four sinusoids. The fitted parameters $a, b$, and $c$ respectively represent the amplitude, frequency, and phase of the decomposed sine (in the yellow box in Figure 10). The 

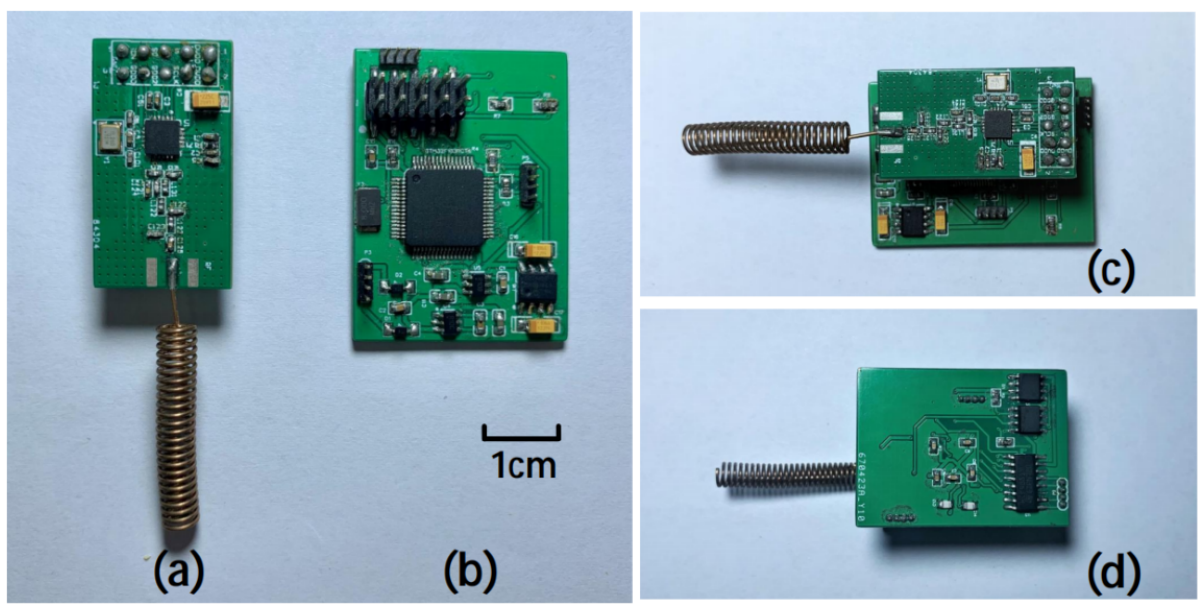

Figure 8. The physical diagrams (a) and (b) of the electronic backpack are the top views of the upper and bottom boards of the electronic backpack, Figure 8(c) is the top view of the two-layer circuit board combined, and Figure $8(\mathrm{~d})$ is the back of the electronic backpack.
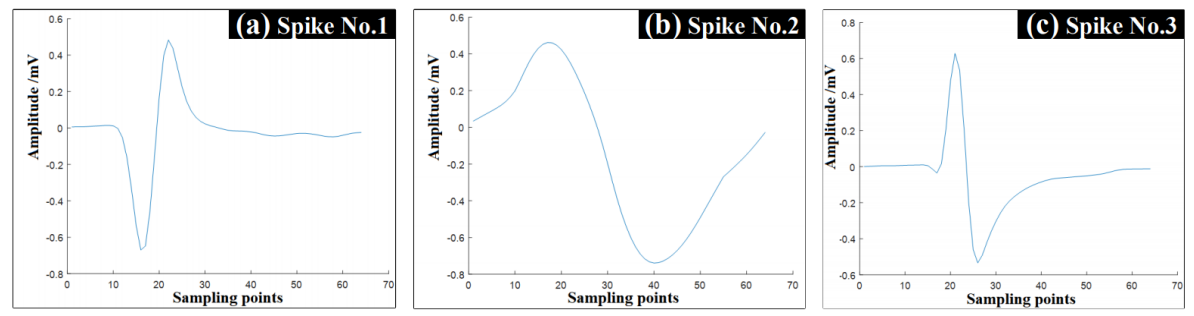

Figure 9. Mean processing result

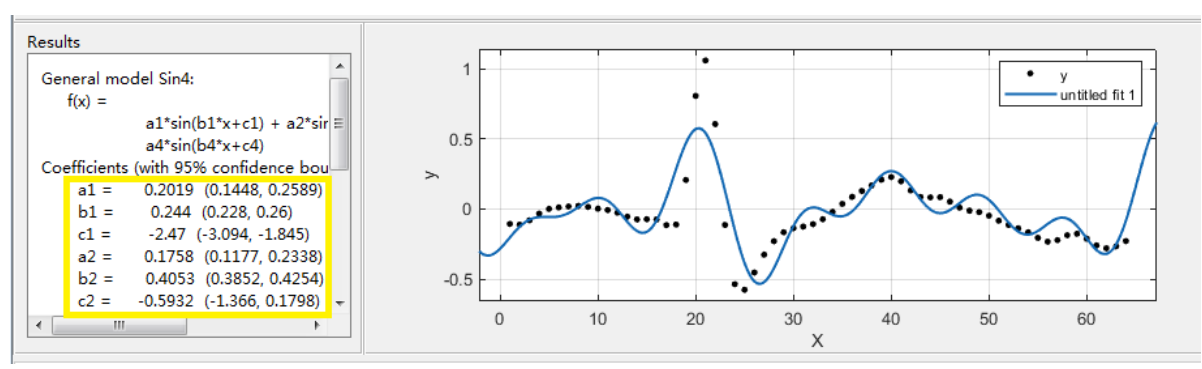

Figure 10. Fitting results

generated fitting parameters are stored in the GUI, and the user can combine and select the desired spike waveform for stimulation in the GUI.

\subsubsection{User graphical interface based on LabVIEW}

The LabVIEW based user interface is the pivotal part of the stimulus analysis system, which mainly completes the functions of stimulus parameter setting, stimulus command sending and stimulus waveform preview. On the one hand, it stores the data parameters processed by MATLAB in the waveform library; on the other hand, it graphically displays and decodes the parameters and transmits them to the transmitting base station. The GUI performs data transmission with the transmitting base station through the USB interface of the computer. After the system is started, the GUI first checks whether the signal transmitting base station has been connected to the computer, and the GUI starts to run after the detection is successful (as shown in the Figure 11). The GUI is developed based on LabVIEW. After testing, the interface can complete the established design requirements. 


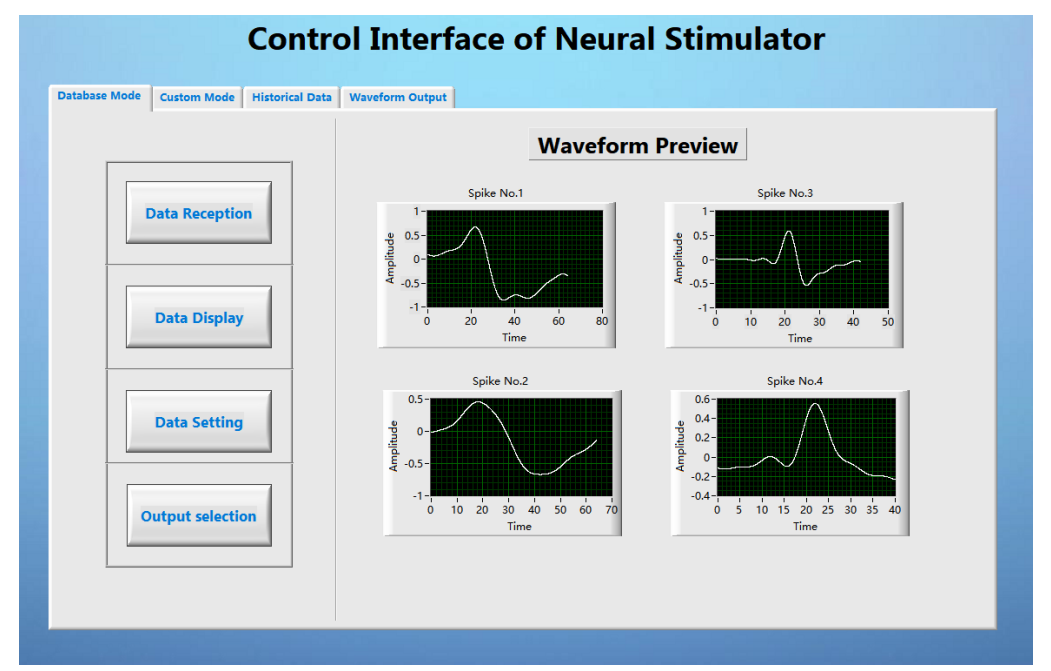

Figure 11. LabVIEW based user interface

\subsubsection{Direct Digital Synthesizer algorithms}

Using the DDS algorithm to generate analog spike signals through DA and amplifiers is the core solution for generating stimulus signals in this paper. DDS technology has been continuously optimized and developed since it was proposed in 1930, and has been widely used in various fields due to its continuous phase, fast conversion speed, and short output frequency interval. Compared with the ordinary look-up table method, the DDS algorithm has higher frequency resolution, more output frequency points, up to $2 \mathrm{Nth}$ frequency points ( $\mathrm{N}$ is the number of bits of the phase accumulator)[31]. Before this, we have decomposed the original spike signal for the synthesis of several sinusoidal signals, the DDS algorithm can be used to simulate the animal's spike signal on the hardware more efficiently.

The basic structure of DDS is divided into 5 parts (as shown in the Figure 12). The first part is the reference clock as the prerequisite for system startup; the core part is the phase accumulator, which controls the output frequency by linearly accumulating the phase value; The third part is the waveform mother table composed of discrete points. The discrete points in the waveform mother table are output as the identification address through the output of the phase accumulator. The remaining two parts are responsible for digital-to-analog conversion and filtering.

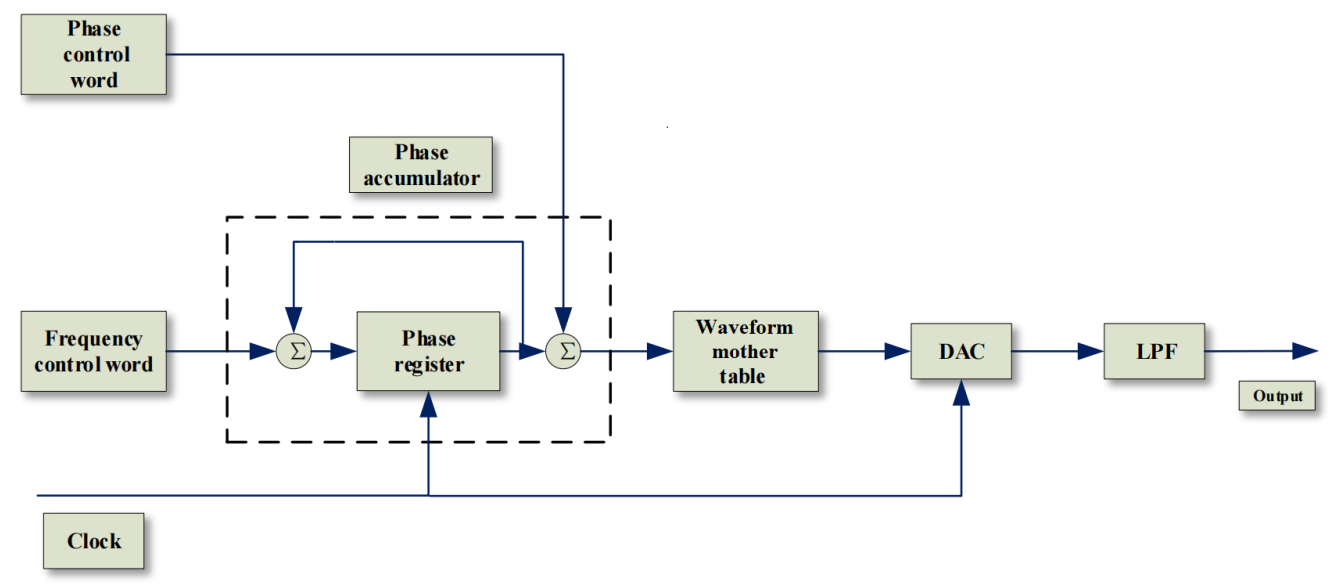

Figure 12. DDS principle block diagram

In the case of low requirements for output frequency and accuracy, the DDS algorithm can also be implemented in the microcontroller. The maximum output frequency of the analog spike signal is $500 \mathrm{~Hz}$. Therefore, a single-chip microcomputer is needed 
as the main control chip to generate analog spike signal. The realization of the DDS principle and the data synthesis of the waveform mother table are the core of the use of the DDS algorithm to synthesize the spike.

The reference clock provides pulses of a specific frequency for the entire system. The phase accumulator accumulates the frequency control word every time it recognizes a pulse. Based on this feature, the counter interrupt is used to simulate the clock, and the phase accumulator is operated on the interrupt. $10 \mathrm{Khz}$ is used as the interrupt frequency of the timer, that is, an interrupt is triggered every $0.1 \mathrm{~ms}$. The phase accumulator can be replaced by a 32-bit data, and the frequency control word is accumulated once for each interruption. By judging the overflow time of the highest bit, it is judged whether the unit cycle is completed. If the phase accumulator is 32 bits, the waveform master table needs 232 values to correspond with it. But in reality, the waveform master table cannot have 232 values. Therefore, use the high 8 -bit of the phase accumulator as the address to query the waveform mother table. In this way, we only need to set an array containing 256 data as the waveform mother table to achieve the goal. The final output frequency is obtained by the following formula:

$$
f_{\text {out }}=M \times \frac{f_{c}}{2^{N}}
$$

Where $M$ is the frequency control word, $f_{c}$ is the clock frequency, and $N$ is the number of bits of the phase accumulator.

The data receiving end receives a section of four sine function parameters that can fit the spike signal. The main control chip at the receiving end first converts the parameters into four sine data tables, and then obtains the wave code table of the analog spike signal through specific operations. The generated wavecode table can be stored as a waveform mother table for output after algorithm processing.

\section{Results}

Before using the stimulator, the effect of system need to be tested. The test environment is shown in the Figure 13.

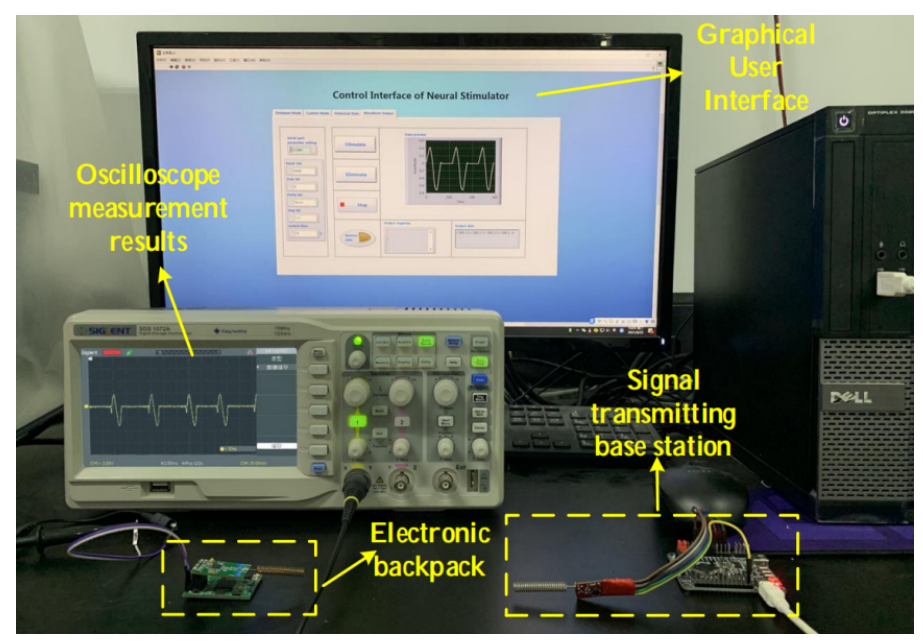

Figure 13. Test environment

\subsection{Stimulus waveform verification}

Since this system is a voltage-type control system, it is only necessary to connect the output terminal to the oscilloscope to test the output waveform. According to system requirements, The GUI can provide multiple stimulation modes, that is, it can control the electronic backpack to generate stimulation sequences of arbitrary waveform 
combinations. The verification of the output waveforms of different stimulus sequences is as follows:

In this system, the user can choose to generate a stimulus sequence containing only one kind of spike (at a fixed time interval). After setting the parameters in the user interface, we connect the electronic backpack to the oscilloscope, and the result is shown in the Figure 14.

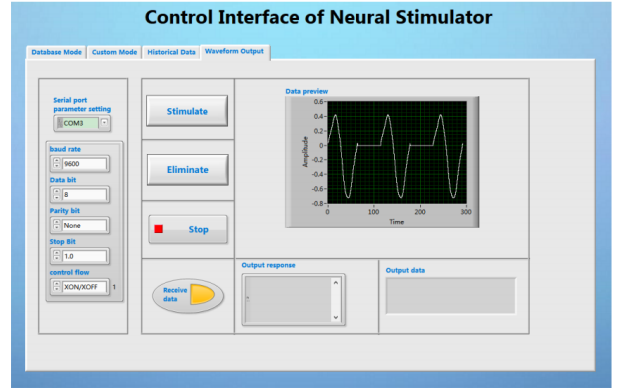

(a)

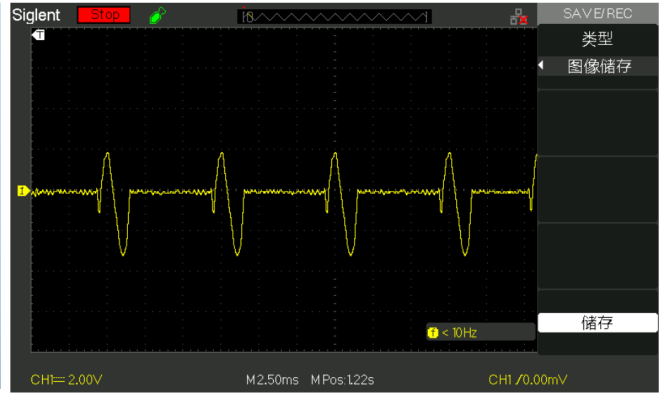

(b)

Figure 14. The comparison diagram of spike output results, (a) is the spike waveform sequence set in the interaction interface, where the spike amplitude is $5 \mathrm{~V}$, the duration is $2 \mathrm{~ms}$, the time interval between spikes is $5 \mathrm{~ms}$, and the entire spike The sequence duration is $500 \mathrm{~ms}$. (b) is the spike output sequence measured by the oscilloscope

The user can also select a stimulus sequence composed of different kinds of spikes, and select up to four kinds of spikes for combination. The frequency, amplitude of the spikes, and the time interval between spikes can all be defined by the user. We chose four different spikes and formed a stimulus sequence for testing. The results are shown in the Figure 15.

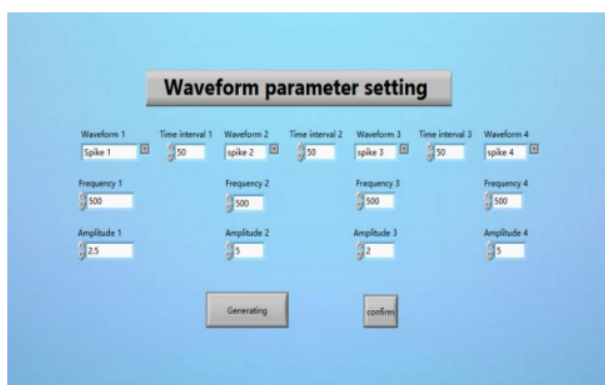

(a)

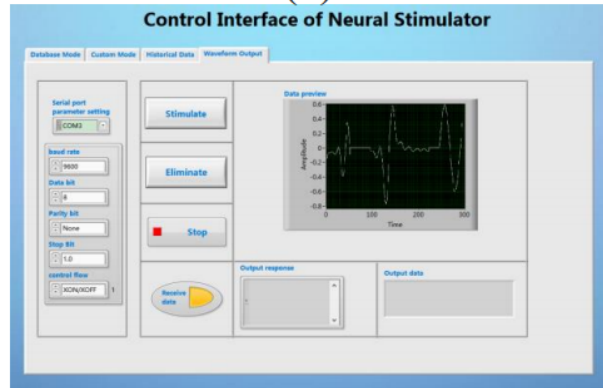

(c)

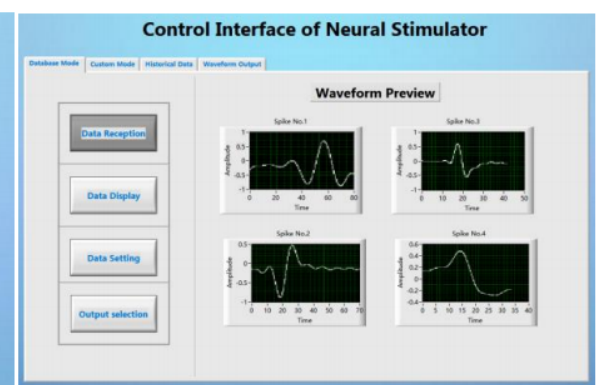

(b)

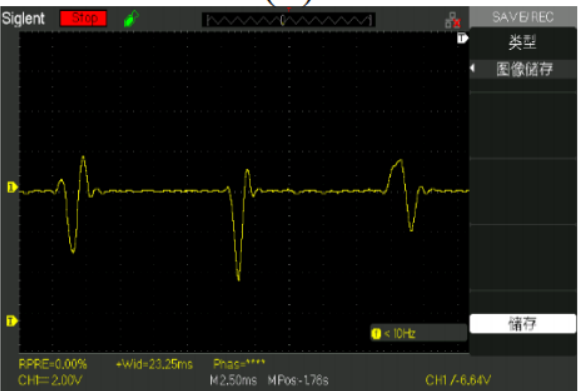

(d)

Figure 15. Spike parameter setting and output verification

The user clicks the Data setting button on the main interface to enter the waveform parameter setting interface sub-vi. In this interface, the user can customize the waveform type and other parameters (as shown in Figure 15(a)). After returning to the main interface and clicking on the waveform playback, the user can extract the currently set waveform (as shown in Figure 15(b)). In the waveform output tab of the main interface, 
the user can preview and output the current stimulus sequence (as shown in Figure 15(c)). After connecting the electronic backpack to the oscilloscope, the parameters of the stimulus sequence we finally tested are consistent with the set waveform parameters (as shown in Figure 15(d)).

In the custom mode, the user can select the stimulation mode in which different waveforms appear alternately. Here we only participate in the test with a $10 \mathrm{~ms}$ interval between a square wave and a sine wave, and a $10 \mathrm{~ms}$ interval between the spike and the square wave. The generated waveform is shown in the Figure 16.
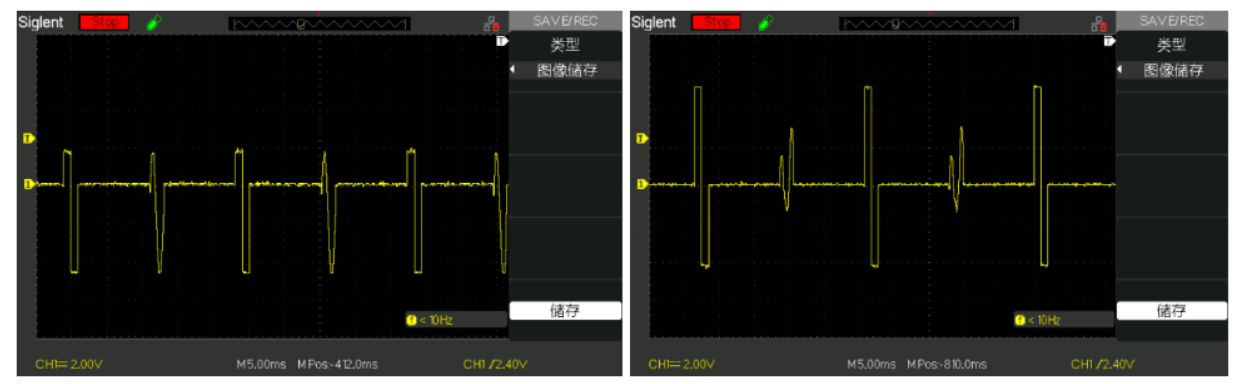

Figure 16. A stimulus sequence with alternating square waves and spikes

The results show that the GUI can well complete functions such as waveform selection, stimulation parameter adjustment, and sending stimulation instructions. At the same time, the hardware part can also complete the spike signal output task well. After the waveform output test is completed and the debugging is correct, we can start animal experiments.

\subsection{Animal experiment}

\subsubsection{Electrode implantation}

In this experiment, microelectrodes are implanted into two motion-related nuclei in the brain: the left and right dorsalis intermedius ventralis anterior (DIVA) nuclei[32]. We use $400 \mathrm{mg} / \mathrm{kg}$ chloral hydrate solution to intramuscularly inject the pigeons and general anesthesia. After the pigeons gradually lose their basic reaction[33], we use $2 \%$ lidocaine hydrochloride injection(including 1/200 000 concentration of epinephrine injection) 0.5 $\mathrm{ml}$ subcutaneous injection in the operation area for local anesthesia[34]. The anesthetized pigeons are fixed on the brain stereotaxic device. The skull is exposed through animal surgery, and hydrogen peroxide with a concentration of $3 \%$ is used for cleaning and disinfection. According to the pigeon brain stereotactic map, the positions of the on the left and right DIVA are located by a three-dimensional stereotaxic instrument. After drilling holes with a skull drill, the electrode is implanted in the corresponding brain area with the help of a stereotaxic device, and the implanted electrode is fixed with medical dental cement. After the dental cement is fully solidified, the lead wire of the electrode is welded to the base, and then the base is fixed on the skull with dental cement. Feed for 5-7 days after the operation, and after observing no physiological abnormalities, it can be used for electrical stimulation experiments.

After the pigeon resumes normal behavior and activities, the electronic backpack is inserted and fixed on the pigeon's head. The output port of the stimulator is connected to the electrode lead-out end, and then the electrical stimulation experiment can be performed. Figure 17 shows a pigeon undergoing electrical stimulation experiments.

\subsubsection{Experimental results}

On the 5th day after the pigeon surgery, the stimulator was used to perform stimulation experiments on the two points of the pigeons, and the most suitable stimulus that can cause the pigeons to react was found. The most suitable stimulus for the two sites is a custom spike combination waveform. The stimulation voltage is $3-5 \mathrm{~V}$, and the duration of each spike signal is $2 \mathrm{~ms}$, and the time interval between the three spikes is 


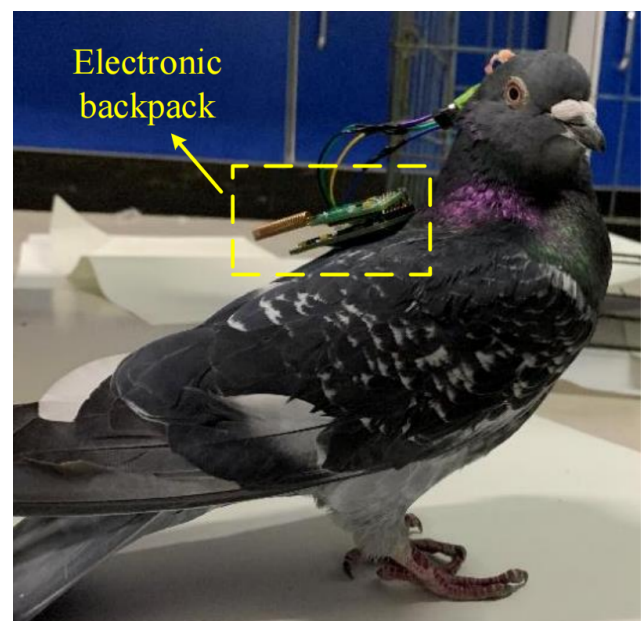

Figure 17. Pigeons undergoing electrical stimulation experiments

$2 \mathrm{~ms}$, that is, every $12 \mathrm{~ms}$ is a stimulation sequence, repeated 40 times, and the stimulation duration is $480 \mathrm{~ms}$ in total. The schematic diagram of the stimulus signal sequence is shown in the Figure 18.

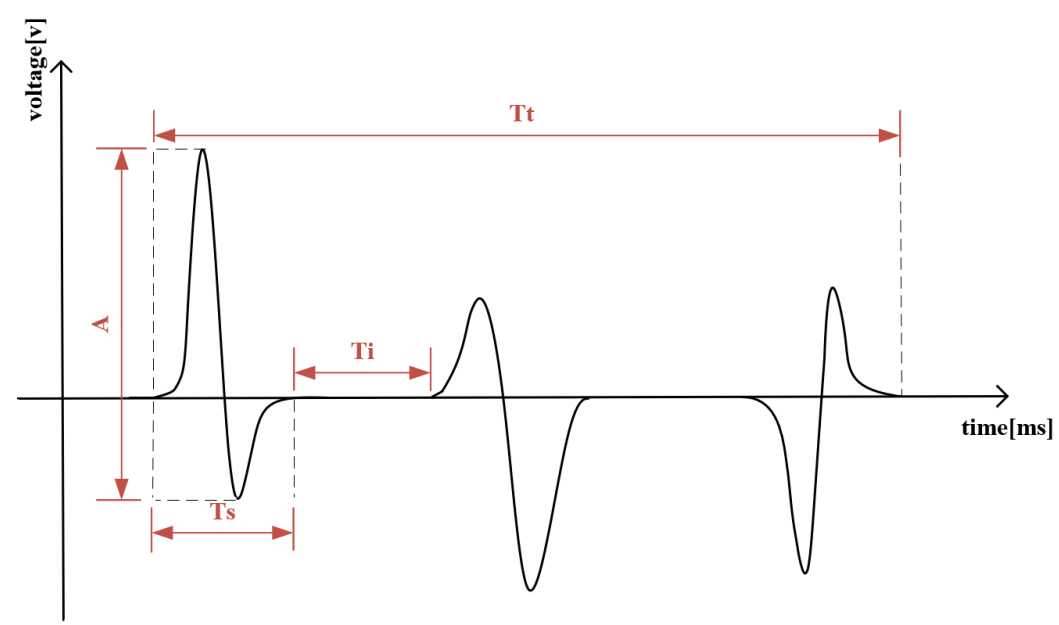

Figure 18. Schematic diagram of the stimulation sequence. $A$ is the signal amplitude, $T_{S}$ is the duration of a single spike signal, $T_{i}$ is the time interval between spikes, and $T_{t}$ is the duration of the entire stimulation sequence

In this paper, 5 pigeons are selected for the experiment, the stimulation is repeated 10 times, and the above-mentioned spike combination waveform is used as the stimulus signal. The results of the experiment are shown in the Table 1. In actual operation, 4 pigeons can basically perform left-right turn reaction according to the control requirements, and the success rate can reach more than $75 \%$. There was 1 animal with a low success rate within the appropriate voltage stimulation range. The reason may be a deviation of the implant position during the electrode implantation or the electrode lead falling off after the pigeon returned to activity.

Table 1. Experimental results of spike stimulation.

\begin{tabular}{cccccc}
\hline Pigeon number & Left turn voltage/V & Number of left turns & right turn voltage/V & Number of right turns & Success rate \\
\hline 1 & 3 & 2 & 3 & 0 & $10 \%$ \\
2 & 3 & 8 & 3 & 7 & $75 \%$ \\
3 & 4 & 9 & 4 & 9 & $90 \%$ \\
4 & 5 & 10 & 4 & 8 & $95 \%$ \\
5 & 4 & 9 & 5 & $8 \%$ \\
\hline
\end{tabular}


The experimental results show that the most suitable stimulation voltage range is $3-5 \mathrm{~V}$ when using the above-mentioned spike stimulation waveform for stimulation. When the voltage is $3 \mathrm{~V}$, the pigeons start to turn left and right. As the voltage increases, the reaction of the pigeons becomes more violent, and the success rate of the left and right turns becomes higher. At this time, the pigeons show obvious behavior of evading and turning.

\section{Conclusion}

In this paper, a wireless animal robot stimulation system based on neuronal electrical signal characteristics is designed. It is no longer limited to using the same waveform for stimulation, but uses analog spikes with spike signal characteristics for stimulation. In order to increase stimulation modes, a mixed form of a variety of stimulation modes are provided, which can be customized to select the spike waveform or the regular waveform to form the desired stimulation sequence. DDS algorithm is used to reconstruct the original spike parameters into analog spike signals to enrich the stimulation waveform in the field of animal robot.

In order to verify the reliability of the system, we used pigeons as the research object to verify the system. From the experimental results, our system is easy to operate and reliable in communication. The communication range can cover every corner of the room, and the communication reliability rate is over $90 \%$. At the same time, the system is small in size and light in weight, and has little impact on the movement of pigeons, which improves the efficiency and success rate of the experiment. In addition, the stimulus signal generated by the system based on the DDS principle to simulate the electrical signal characteristics of the neuron. It successfully caused the pigeon's stress response. Indicating that the simulation of the action potential signal of the animal's neuron as a stimulus signal is feasible in the control of animal robots. At the same time, the multi-modal stimulation used by this system breaks the traditional stimulation method and increases the stimulation mode of the animal robot. This stimulus method provides effective ideas and methods for solving the problem of "stimulation fatigue" in the future.

In the future, we hope to continue to optimize this system, such as adding a camera function. Our system can only control the pigeon's motion behavior through visual judgment, and the control range is limited. If a miniature camera is added to control behavior through feedback pictures, the application range of the pigeon robot will be greatly increased. At the same time, how to quantify the degree of damage to animal brain tissue caused by different stimulation waveforms is a problem and direction that we need to consider in the future.

Author Contributions: Conceptualization, R.Y. and J.Y.; methodology, R.Y. and Z.C.; software, R.Y. and Y.L; hardware, R.Y., Z.C. and Y.S.; experimental test, R.Y. , Z.C and Q.W; writing-original draft preparation, R.Y.; writing - review and editing,R.Y. and H.W.; project administration, R.H.; funding acquisition, J.Y. and H.W. All authors have read and agreed to the published version of the manuscript.

Institutional Review Board Statement: The study was conducted according to the guidelines of the Declaration of Helsinki, and approved by the Medical Ethics Committee of Affiliated Hospital of Qingdao University(protocol code: AHQU202103150073 and date of approval:March 15, 2020).

Funding: This research was funded by the National Natural Science Foundation of China(61903230), Natural Science Foundation of Shandong Province(ZR2020MF098), the Taishan Scholar Project of Shandong Province of China.

Conflicts of Interest: The authors declare no conflict of interest.

\section{References}

1. Zhou,Z.; Liu,D.;Sun,H. Pigeon Robot for Navigation Guided by Remote Control: System Construction and Functional Verification. Journal of Bionic Engineering 2021, 18(1), 184-196. 
2. Sato,H.; Berry,W.; Casey,E. A cyborg beetle: Insect flight control through an implantable, tetherless microsystem. International Conference on Micro Electro Mechanical Systems 2008, 164-167.

3. Nguyen,D.; Tan,Z.; Sato,H. Sideways Walking Control of a Cyborg Beetle. Medical Robotics and Bionics 2020, 2(3), 311-337.

4. Jia,Y.; Guler,U.; Lai,Y.P.; Gong, Y.; Weber, A.; Li, W.; Ghovanloo, M. A Trimodal Wireless Implantable Neural Interface System-on-Chip. In Proceedings of the 2020 IEEE International Solid-State Circuits Conference-(ISSCC), San Francisco, CA, USA, 16-20 February 2020, pp, 323-330.

5. Edward,E.S.;Kouzani,A.Z.; Tye,S.J. Towards miniaturized closed-loop optogenetic stimulation devices. Neural Eng 2018, 15, 021002.

6. Fuchs,E.; Holmes,P.; Kiemel,T. Intersegmental Coordination of Cockroach Locomotion: Adaptive Control of Centrally Coupled Pattern Generator Circuits. Frontiers in Neural Circuits 2011, 4,125.

7. Brown,S. Stealth sharks to patrol the high seas. New Scientist magazine 2006, 2541:30.

8. Su,X.C.;Huai,R.T.; Yang,J.Q.; Wang,H. Brain mechanism and methods for robo-animal motor behavior control. Sci. Sin 2012,4,1130-1146.

9. Kobayashi,N.; Yoshida,M.;Matsumoto,N Artificial control of swimming in goldfish by brain stimulation: Confirmation of the midbrain nuclei as the swimming center. Neuroscience Letters 2009, 452( 1), 42-46.

10. Erickson,J.C.; Herrera,M, Bustamante,M. Effective stimulus parameters for directed locomotion in Madagascar hissing cockroach biobot. PloS one 2015, 10(8), e0134348.

11. Kobayashi,N.;Yoshida,M.;Matsumoto,N Artificial control of swimming in goldfish by brain stimulation: Confirmation of the midbrain nuclei as the swimming center. Neuroscience Letters 2009,10(8), 42-46.

12. Shim,S.; Yun,S.; Kim,S. A handheld neural stimulation controller for avian navigation guided by remote control Bio-medical materials and engineering 2019,30(5-6),497-507

13. Yun,S.; Koh,C.S.; Jeong,J. Remote-controlled fully implantable neural stimulator for freely moving small animal. Electronics 2019,8(6), 706.

14. Seo,J.; Choi,J.; Park,S.; et al. Wireless navigation of pigeons using polyme r-based fully implantable stimulator: a pilot study using depth electrodes. Engineering in Medicine and Biology Society 2017, 917-920.

15. Lin,Y.J.; Lee,S.Y. A Microstimulator with Parameter Adjustment for Bladder Dysfunction. 2017 IEEE International Symposium on Circuits and Systems (ISCAS).MD, USA, 28-31 May 2017.

16. Lee,B.; Koripalli,M.K.; Jia,Y.; Acosta,J.; Sendi, M.S.E.; Choi, Y.; Ghovanloo, M.. Wireless navigation of pigeons using polyme r-based fully implantable stimulator: a pilot study using depth electrodes. Engineering in Medicine and Biology Society 2017, 917-920.

17. Seo,J.; Wee,J.H.; Park,J.H.; Park,P.; Kim,J.-W.; Kim,S.J. Nerve cuffff electrode using embedded magnets and its application to hypoglossal nerve stimulation. Neural Eng 2016, 13, 066014.

18. Potter, K. A.; Buck, A. C.; Self, W. K. Capadona, J. R. Stab injury and device implantation within the brain results in inversely multiphasic neuroinflammatory and neurodegenerative responses. Journal of Neural Engineering 2012, 9, 046020.

19. Chestek, C. A. Long-term stability of neural prosthetic control signals from silicon cortical arrays in rhesus macaque motor cortex. Journal of Neural Engineering 2011,8, 045005.

20. Kozai,T.D.et al. Ultrasmall implantable composite microelectrodes with bioactive surfaces for chronic neural interfaces. Nature Materials 2012, 11,1065-1073.

21. Sanchez,J.C.; Alba,N.; Nishida,T.; Batich,C. Carney, P. R. Structural Modifications in Chronic.Microwire Electrodes for Cortical Neuroprosthetics: A Case Study. IEEE Transactions on Neural Systems and Rehabilitation Engineering 2006, 14, 217-221.

22. International Symposium ELMAR-2006 of the IEEE, 2006, pp. 259-262.

23. Purcell,E.K.; Thompson, D. E.; Ludwig, K. A. ; Kipke, D. R. Flavopiridol reduces the impedance of neural prostheses in vivo without affecting recording quality. Journal of neuroscience methods 2009, 183,149-157.

24. Jia, Y.; Mirbozorgi, S.A.; Lee, B.; Khan, W.; Madi, F.; Inan, O.T.; Weber,A.; Li, W.; Ghovanloo, M. A mm-sized free-floating wirelessly powered implantable optical stimulation device. IEEE Trans. Biomed. Circuits Syst 2019, 13, 608-618. 
25. Elsherbini,A.; Wu,J.F.; Sarabandi,K. Dual Polarized Wideband Directional Coupled Sectorial Loop Antennas for Radar and Mobile Base Station Applications. IEEE Transactions on Antennas and Propagation 2015, 63(4), 1505-1513.

26. Tripathi,S.; Mohan,A.; Yadav,S. A Compact Koch Fractal UWB MIMO Antenna With WLAN Band-Rejection. IEEE Antennas and Wireless Propagation Letters 2015, 14, 15651568.

27. Wen-Ao,L.;Zhi-Hong,T.;Qing-Xin,C.; Xioao-Hu,Wu. Differential Stepped-Slot UWB Antenna With Common-Mode Suppression and Dual Sharp-Selectivity Notched Bands. IEEE Antennas and Wireless Propag.Lett 2016.

28. SHennig,H.; Hurwitz,C.; Sorbaro,M. Scaling Spike Detection and Sorting for NextGeneration Electrophysiology. Advances in neurobiology 2019, 22, 171-184.

29. [29]E. A. Vallicelli, et al. (2017, Oct). "Neural Spikes Digital Detector/Sorting on FPGA". In Biomedical Circuits and Systems (BioCAS), 2017 IEEE International Conference on (pp. 1-4). IEEE.

30. Vallicelli, E.A.; Fary, F.; Baschirotto, A.; de Matteis, M.; Reato, M.; Maschietto, M.; Rocchi, F.; Vassanelli, S.; Guarrera, D.; Collazuol, G. BReal-Time Digital Implementation of a Principal Component Analysis Algorithm for Neurons Spike Detection. In Proceedings of the 2018 International Conference on IC Design and Technology (ICICDT), Otranto, Italy, 4-6 June 2018; pp. 33-36.

31. Chen,L.; Sun Q.; Zhao Y. Design and experiments of a waveform generator based on DDS technology. Recent Patents on Engineering 2020, 14(4), 588-597.

32. Karten,H. J.; Konishi,M.; Pettigrew,J. D. Somatosensory representation in the anteriorwulst of the owl (Speotytocunicularia). Neurosci Abstr 1987, 5, 554.

33. Wild,J. M. The avain somatosensory system: connections of regions of body representation in the forebrain of the pigeon.Brain Res 1987, 412, 205-223.

34. Schneider,A.; Necker,R. Electrophysiological investigations of the somatosensory thalamus of the pigeon. Exp Brain Res 1996, 109, 377-383. 
16 of 14 